\title{
Probing loop effects in wrong-sign Yukawa coupling region of Type-II 2HDM
}

\author{
Wei $\mathrm{Su}^{\mathrm{a}}$ \\ ARC Centre of Excellence for Dark Matter Particle Physics, Department of Physics, University of Adelaide, Adelaide, South Australia 5005, \\ Australia
}

Received: 18 April 2020 / Accepted: 2 May 2021 / Published online: 10 May 2021

(C) The Author(s) 2021

\begin{abstract}
In the framework of 2HDM, we explore the wrong-sign Yukawa region with direct and indirect searches up to one-loop level. The direct searches include the latest $H / A \rightarrow f \bar{f}, V V, V h, h h$ reports at current LHC, and the study of indirect Higgs precision measurements works with current LHC, future HL-LHC and CEPC. At tree level of Type-II 2HDM, for degenerate heavy Higgs mass $m_{A}=$ $m_{H}=m_{H^{ \pm}}<800 \mathrm{GeV}$, the wrong-sign Yukawa regions are excluded largely except for the tiny allowed region around $\cos (\beta-\alpha) \in(0.2,0.3)$ under the combined Higgs constraints. The excluded region is also nearly independent of parameter $m_{12}$ or $\lambda v^{2}=m_{A}^{2}-m_{12}^{2} /(\sin \beta \cos \beta)$. The situation changes a lot after including loop corrections to the indirect searches, for example $m_{A}=1500 \mathrm{GeV}$, the region with $\lambda v^{2}<0$ will be stronger constrained to be totally excluded. Whilst parameter space with $\lambda v^{2}>0$ would get larger survived wrong-sign region for $m_{A}=800 \mathrm{GeV}$ compared to it at tree level. We also conclude Higgs direct searches works better on constraining $\lambda v^{2} \approx 0 \mathrm{GeV}$ range than theoretical constraints. We also find that the loop-level wrong-sign Yukawa limit only occurs at mass decoupling scale.
\end{abstract}

\section{Contents}

1 Introduction and motivation $\ldots \ldots \ldots \ldots$

2 Two Higgs doublet models . . . . . . . . . . . . . 2

2.1 2HDM Higgs sector . . . . . . . . . . . . 2

2.2 Wrong-sign Yukawa of $2 \mathrm{HDM} \ldots \ldots . . .2$

3 Study method . . . . . . . . . . . . . . . . 3

4 Results at tree level . . . . . . . . . . . . . . . 4

4.1 Indirect search at LHC and future colliders . . 4

4.2 Wrong-sign region and disappeared up-type . . 4

4.3 Current LHC direct search . . . . . . . . . . 5

5 Results at one-loop level . . . . . . . . . . 7

a e-mail: wei.su@ adelaide.edu.au (corresponding author)
5.1 Loop effects in $\cos (\beta-\alpha)-\tan \beta$ plane . . 7

5.2 Loop effects in $m_{\Phi}-m_{12}$ plane . . . . . . 9

6 Conclusions ................. . 9

References . . . . . . . . . . . . . . . . . 10

\section{Introduction and motivation}

Since the discovery of Standard Model (SM) -like Higgs boson at LHC Run-I [1,2], SM is confirmed to be one selfconsistent theory, and exploring Higgs boson properties especially Higgs couplings becomes a promising window to study new physics beyond-the-SM (BSM). Meanwhile motivated by various experimental and theoretical hits, to extend SM Higgs sector becomes necessary to address them.

Among numerous extensions, Two Higgs Doublet Model (2HDM) is a well motivated framework [3-6]. After electroweak symmetry breaking (EWSB), the general 2HDM will generate 5 mass eigenstates, a pair of charged Higgs $H^{ \pm}$, one CP-odd Higgs boson $A$ and two CP-even Higgs bosons, $h, H$. Here we take the lighter $h$ as the measured SM-like Higgs.

Since the improvements of various experiments, the wrong-sign region have attracted fruitful researches [716]. This work focuses on testing the so-called wrong-sign Yukawa region up to one-loop level with both indirect and direct searches at current LHC. For the direct searches, we constrain the parameter space with various heavy Higgs decays, taking the cross section times branching ratio $\sigma \times \mathrm{Br}$ limits of various channels, including $A / H \rightarrow \mu \mu$ [17-19], $A / H \rightarrow b b$ [20,21], $A / H \rightarrow \tau \tau$ [22-24], $A / H \rightarrow t t$ [25,26], $H \rightarrow Z Z[27,28], H \rightarrow W W[29,30]$ at tree level. For the indirect searches, we perform the global fit the SM-like Higgs precision measurement from LHC Run-II [31], HL-LHC [32] and CPEC [33] up to one-loop level. The results show that the wrong-sign Yukawa region for $m_{A}<800 \mathrm{GeV}$ is strongly constrained. But the constraints 
get weaker afer including the loop correction to Higgs precision studies for $\lambda v^{2}>0$. While for $m_{A}=1500 \mathrm{GeV}$ with $\lambda v^{2}>0$, the constraints get stronger compared to it at tree level.

Our paper is structured as follows. In Sect. 2, we will give a brief introduction to 2HDMs, concentrated on the wrongsign Yukawa analysis. We give a brief summary of study methods and the relevant experimental reports in Sect. 3 . Then at Sects. 4 and 5 we present our analyses and results at tree and one-loop level respectively. Finally we will give our main conclusions in Sect. 6.

\section{Two Higgs doublet models}

\subsection{HDM Higgs sector}

The general 2HDM has two $\mathrm{SU}(2)_{L}$ scalar doublets $\Phi_{i}(i=$ $1,2)$ with hyper-charge $Y=+1 / 2$,

$\Phi_{i}=\left(\begin{array}{c}\phi_{i}^{+} \\ \left(v_{i}+\phi_{i}^{0}+i G_{i}\right) / \sqrt{2}\end{array}\right)$.

where $v_{i}(i=1,2)$ are the vacuum expectation values (vev) of the two doublets after EWSB with $v_{1}^{2}+v_{2}^{2}=v^{2}=$ $(246 \mathrm{GeV})^{2}$ and $\tan \beta=v_{2} / v_{1}$.

The 2HDM Lagrangian for the Higgs sector can be written as

$\mathcal{L}=\sum_{i}\left|D_{\mu} \Phi_{i}\right|^{2}-V\left(\Phi_{1}, \Phi_{2}\right)+\mathcal{L}_{\text {Yuk }}$,

with a Higgs potential of

$$
\begin{aligned}
V\left(\Phi_{1}, \Phi_{2}\right)= & m_{11}^{2} \Phi_{1}^{\dagger} \Phi_{1}+m_{22}^{2} \Phi_{2}^{\dagger} \Phi_{2}-m_{12}^{2}\left(\Phi_{1}^{\dagger} \Phi_{2}+\text { h.c. }\right) \\
& +\frac{\lambda_{1}}{2}\left(\Phi_{1}^{\dagger} \Phi_{1}\right)^{2}+\frac{\lambda_{2}}{2}\left(\Phi_{2}^{\dagger} \Phi_{2}\right)^{2} \\
& +\lambda_{3}\left(\Phi_{1}^{\dagger} \Phi_{1}\right)\left(\Phi_{2}^{\dagger} \Phi_{2}\right)+\lambda_{4}\left(\Phi_{1}^{\dagger} \Phi_{2}\right)\left(\Phi_{2}^{\dagger} \Phi_{1}\right) \\
& +\frac{\lambda_{5}}{2}\left[\left(\Phi_{1}^{\dagger} \Phi_{2}\right)^{2}+\text { h.c. }\right],
\end{aligned}
$$

where we have assumed $C P$ conservation, and a soft $\mathbb{Z}_{2}$ symmetry breaking term $m_{12}^{2}$. For the neutral CP-even Higgs, with $\alpha$ as the rotation angle diagonalizing the CP-even Higgs mass matrix,

Table 1 Interactions between fermions and Higgs doublets in four types of $2 \mathrm{HDM}$

\begin{tabular}{llll}
\hline & Up-type & Down-type & Lepton \\
\hline Type-I & $\Phi_{1}$ & $\Phi_{1}$ & $\Phi_{1}$ \\
Type-II & $\Phi_{1}$ & $\Phi_{2}$ & $\Phi_{2}$ \\
Type-LS & $\Phi_{1}$ & $\Phi_{1}$ & $\Phi_{2}$ \\
Type-F & $\Phi_{1}$ & $\Phi_{2}$ & $\Phi_{1}$ \\
\hline
\end{tabular}

$\left(\begin{array}{c}H \\ h\end{array}\right)=\left(\begin{array}{cc}\cos \alpha & \sin \alpha \\ -\sin \alpha & \cos \alpha\end{array}\right)\left(\begin{array}{l}\phi_{1}^{0} \\ \phi_{2}^{0}\end{array}\right)$,

In this work we set $m_{H}>m_{h}=125 \mathrm{GeV}$, and by convention, here we set $0 \leq \beta \leq \frac{\pi}{2}, 0 \leq \beta-\alpha \leq \pi$. The most general Yukawa interactions of $\Phi_{1,2}$ with the SM fermions under the $Z_{2}$ symmetry is

$$
\begin{aligned}
-\mathcal{L}_{Y u k}= & Y_{u} \bar{Q}_{L} i \sigma_{2} \Phi_{u}^{*} u_{R} \\
& +Y_{d} \bar{Q}_{L} \Phi_{d} d_{R}+Y_{e} \bar{L}_{L} \Phi_{e} e_{R}+\text { h.c. }
\end{aligned}
$$

where $\Phi_{u, d, e}$ are either $\Phi_{1}$ or $\Phi_{2}$. Depending on the interactions of $\Phi_{i}$ coupling to the fermion sector, there are typically four types of 2HDM (Table 1):

For a review on different types of $2 \mathrm{HDM}$ as well as the phenomena, see Ref. [34]. Table 2 is Higgs couplings to the SM fermions in the four different types of 2HDM, normalized to the corresponding SM values, for a better analysis at following sections.

In the following sections, we will take $\kappa_{x}=\kappa_{h}^{x}$. For normalized SM-like Higgs gauge couplings, $V=Z, W^{ \pm}$,

$\kappa_{V} \equiv \frac{g_{\mathrm{hVV}}^{2 \mathrm{HDM}}}{g_{\mathrm{hVV}}^{\mathrm{SM}}}=\sin (\beta-\alpha)$

with $\operatorname{sign}\left(\kappa_{V}\right)=1$ by convention.

After EWSB, three Goldstone bosons are absorbed by the SM gauge bosons $Z, W^{ \pm}$, providing their masses. The remaining physical mass eigenstates are $h, H, A$ and $H^{ \pm}$. Instead of the eight parameters appearing in the Higgs potential $m_{11}^{2}, m_{22}^{2}, m_{12}^{2}, \lambda_{1,2,3,4,5}$, a more convenient choice of the parameters is $v, \tan \beta, \alpha, m_{h}, m_{H}, m_{A}, m_{H^{ \pm}}, m_{12}^{2}$.

\subsection{Wrong-sign Yukawa of 2HDM}

Taking the notations in [35], we define,

$$
\begin{aligned}
\kappa_{U} \equiv \frac{\cos \alpha}{\sin \beta}= & 1+\cos (\beta-\alpha) \cot \beta-\frac{1}{2} \cos ^{2}(\beta-\alpha) \\
& +\mathcal{O}\left(\cos ^{2}(\beta-\alpha)\right) \\
\kappa_{D} \equiv-\frac{\sin \alpha}{\cos \beta}= & 1-\cos (\beta-\alpha) \tan \beta-\frac{1}{2} \cos ^{2}(\beta-\alpha) \\
& +\mathcal{O}\left(\cos ^{2}(\beta-\alpha)\right)
\end{aligned}
$$

When $\sin (\beta-\alpha)=1$, all the SM-like Higgs boson couplings in four types will be exact same as them in SM respectively, which is the usual case called as alignment limit. These terms also can be written in the other mode,

$$
\begin{aligned}
\kappa_{U}= & \sin (\beta+\alpha)+\cos (\beta+\alpha) \cot \beta \\
= & \pm 1+\cos (\beta+\alpha) \cot \beta \mp \frac{1}{2} \cos ^{2}(\beta+\alpha) \\
& +\mathcal{O}\left(\cos ^{2}(\beta+\alpha)\right) \\
\kappa_{D}= & -\sin (\beta+\alpha)+\cos (\beta+\alpha) \tan \beta \\
= & \mp 1+\cos (\beta+\alpha) \tan \beta \pm \frac{1}{2} \cos ^{2}(\beta+\alpha)
\end{aligned}
$$


Table 2 Higgs couplings to the SM fermions in the four different types of 2HDM, normalized to the corresponding SM values

\begin{tabular}{llllllllcc}
\hline & \multicolumn{1}{l}{ Normalized Higgs couplings } \\
\cline { 2 - 7 } & $\kappa_{h}^{u}$ & $\kappa_{h}^{d}$ & $\kappa_{h}^{e}$ & $\kappa_{H}^{u}$ & $\kappa_{H}^{d}$ & $\kappa_{H}^{e}$ & $\kappa_{A}^{u}$ & $\kappa_{A}^{d}$ \\
\hline Type-I & $\frac{\cos \alpha}{\sin \beta}$ & $\frac{\cos \alpha}{\sin \beta}$ & $\frac{\cos \alpha}{\sin \beta}$ & $\frac{\sin \alpha}{\sin \beta}$ & $\frac{\sin \alpha}{\sin \beta}$ & $\frac{\sin \alpha}{\sin \beta}$ & $\cot \beta$ & $-\cot \beta$ & $-\cot \beta$ \\
Type-II & $\frac{\cos \alpha}{\sin \beta}$ & $-\frac{\sin \alpha}{\cos \beta}$ & $-\frac{\sin \alpha}{\cos \beta}$ & $\frac{\sin \alpha}{\sin \beta}$ & $\frac{\cos \alpha}{\cos \beta}$ & $\frac{\cos \alpha}{\cos \beta}$ & $\cot \beta$ & $\tan \beta$ & $\tan \beta$ \\
Type-LS & $\frac{\cos \alpha}{\sin \beta}$ & $\frac{\cos \alpha}{\sin \beta}$ & $-\frac{\sin \alpha}{\cos \beta}$ & $\frac{\sin \alpha}{\sin \beta}$ & $\frac{\sin \alpha}{\sin \beta}$ & $\frac{\cos \alpha}{\cos \beta}$ & $\cot \beta$ & $-\cot \beta$ & $\tan \beta$ \\
Type-F & $\frac{\cos \alpha}{\sin \beta}$ & $-\frac{\sin \alpha}{\cos \beta}$ & $\frac{\cos \alpha}{\sin \beta}$ & $\frac{\sin \alpha}{\sin \beta}$ & $\frac{\cos \alpha}{\cos \beta}$ & $\frac{\sin \alpha}{\sin \beta}$ & $\cot \beta$ & $\tan \beta$ & $-\cot \beta$ \\
\hline
\end{tabular}

$$
+\mathcal{O}\left(\cos ^{2}(\beta+\alpha)\right)
$$

Here we can get $\sin (\beta+\alpha)=1, \kappa_{U}=-\kappa_{D}=1$, whilst $\sin (\beta+\alpha)=-1, \kappa_{U}=-\kappa_{D}=-1$, which is usually called "wrong-sign" Yukawa limit in 2HDM.

Wrong-sign Yukawa Regime As defined in [35], the wrong-sign Yukawa regime requires at least one of sign of Yukawa couplings is opposite to Higgs vector boson coupling, in physics which can be expressed as,

$$
\begin{gathered}
\operatorname{sign}\left(g_{U / D}^{2 \mathrm{HDM}}\right) \operatorname{sign}\left(g_{V}^{2 \mathrm{HDM}}\right)=-1 \\
\text { With } \kappa_{i} \equiv g_{i}^{2 \mathrm{HDM}} / g_{i}^{\mathrm{SM}}, \text { it is, } \\
\operatorname{sign}\left(\kappa_{U / D}\right) \operatorname{sign}\left(\kappa_{V}\right)=-1 \\
\text { or } \quad \kappa_{U / D} \kappa_{V}<0
\end{gathered}
$$

for any up-type or down-type quark. Physically this definition is suitable for both tree- and loop-level study.

For the gauge couplings $\kappa_{V}=\sin (\beta-\alpha)$, it is always positive in our notaion. Through Table 2, Type-I 2HDM only has the wrong $\kappa_{U}=-1$ case, and other three types would have both $\kappa_{U}=-1$ or $\kappa_{D}=-1$ cases. It would deviate from 1 significantly, which could be one important constraint for parameter space of the wrong-sign Yukawa region.

But even at future lepton colliders, the wrong-sign Yukawa region at tree level will be allowed as shown in Fig. 3, even the allowed $|\cos (\beta-\alpha)|$ is less than 0.007 . This situation can be changed once the loop level corrections are included,

$\kappa_{U}^{\text {loop }}=\kappa_{U}+\Delta_{U}^{\text {loop }}, \kappa_{D}^{\text {loop }}=\kappa_{D}+\Delta_{D}^{\text {loop }}$

Here $\Delta_{U}^{\text {loop }}, \Delta_{D}^{\text {loop }}$ are loop corrections, dependent on all parameters $\alpha, \beta, m_{12}$ and four Higgs masses. $\left|\kappa_{U}^{\text {loop }}\right|$ and $\left|\kappa_{D}^{\text {loop }}\right|$ would not be exact 1 at same time until the decoupling effect comes.

\section{Wrong-sign Yukawa Limit}

For Type-II,

$\kappa_{D}=-1, \kappa_{U}=1$

This definition, wrong-sign limit, works for both tree- and one-loop level studies.

At tree level, from Eqs. (9) and (10), $\sin (\beta+\alpha)=$ $1, \cos (\beta+\alpha)=0$ is the limit.
At one-loop level, to reach at wrong-sign limit at Eq. (13), mass decoupling and $\sin (\beta+\alpha)=1, \cos (\beta+\alpha)=0$ are all in need. At this limit, $\Delta_{U / D}^{\text {loop }}$ are negligible and all values become same as them at tree level. Under current measurements, there are allowed regions deviated from this exact limit at loop level.

In this work, we will address one-loop level $\Delta^{1-\text { loop }}$ effects to the global fit results around wrong-sign Yukawa region before the decoupling scale, with Higgs precision measurement at current LHC Run-II and future HL-LHC, CEPC.

\section{Study method}

Since the discovery of $125 \mathrm{GeV}$ Higgs boson at LHC Run-I, the study of Higgs sector, both the SM-like Higgs boson precision measurements and direct search of additional Higgs boson, has fruitful results. To have a complete study of wrong-sign Yukawa region of 2HDM, here we will explore its properties with both direct and indirect experimental reports at LHC Run-II.

To interpret the experimental direct search reports, we take the cross section times branching ratio $\sigma \times \mathrm{Br}$ limits of various channels, including $A / H \rightarrow \mu \mu$ [17-19], $A / H \rightarrow b b[20,21], A / H \rightarrow \tau \tau$ [22-24], A/H $\rightarrow t t$ [25,26], $H \rightarrow Z Z[27,28], H \rightarrow W W[29,30]$. About the theoretical predictions in the 2HDM parameter space, we get $\sigma \times B r$ with the SusHi package [36] for the production cross-section at NNLO level, and 2HDMC [37] code for Higgs decay branching ratio at tree level.

About the indirect search, we transfer the errors of SMlike Higgs boson couplings to the constraints on the model parameters at one-loop level, adopting the on-shell renormalization scheme [38] for Higgs masses, $\alpha, \beta$, vacuum expectation value $v$, and minimal substraction scheme for parameter $m_{12}$. The conventions for the renormalization constants, and conditions follows Refs. [38,39], which are two-point functions of Higgs field. More details are discussed at our previous work [40], and our numerical results keeps consistent with package $\mathrm{H}$-coup [41]. We make a global fit by constructing 
the $\chi^{2}$ with the profile likelihood method

$\chi^{2}=\sum_{i} \frac{\left(\mu_{i}^{\mathrm{BSM}}-\mu_{i}^{\mathrm{obs}}\right)^{2}}{\sigma_{\mu_{i}}^{2}}$.

Here $\mu_{i}^{\mathrm{BSM}}=\frac{(\sigma \times \mathrm{Br})_{\mathrm{BSM}}}{(\sigma \times \mathrm{Br})_{\mathrm{SM}}}$ for various Higgs search channels and $\sigma_{\mu_{i}}$ is the experimental precision on a particular channel. $\mu_{i}^{\mathrm{BSM}}$ is predicted in each specific model, depending on model parameters. For the LHC Run-II, the measured $\mu_{i}^{\text {obs }}$ and corresponding $\sigma_{\mu_{i}}$ are given by ATLAS at $13 \mathrm{TeV}$ up to $80 \mathrm{fb}^{-1}$ [31]. In our analyses of the future colliders, $\mu_{i}^{\text {obs }}$ are set to be the SM value: $\mu_{i}^{\text {obs }}=1$, assuming no deviation to the SM observables are observed. For the corresponding $\sigma_{\mu_{i}}$ of the HL-LHC and CEPC, we take the precision measurements from [32,33]. The future FCC-ee [42] has similar performance to CEPC [43], thus here we will only show the results with CEPC. For one or two parameter fit, the corresponding $\Delta \chi^{2}=\chi^{2}-\chi_{\min }^{2}$ for $95 \%$ C.L. is 3.84 or 5.99, respectively.

In 2HDMs, the additional Higgs sector involves several Higgs self-couplings, which are constrained by various theories considerations, such as vacuum stability, perturbativity and unitarity. For the detailed study, we refer to the results in works $[40,43]$. The general idea is $-(125 \mathrm{GeV})^{2} \leq \sqrt{\lambda v^{2}} \leq$ $(600 \mathrm{GeV})^{2}$, and we will study inside of this region.

\section{Results at tree level}

Based on the discussion above, first we will show our study results at tree level. It includes the current LHC direct and indirect searches, as well as the indirect searches at future HL-LHC and CEPC.

\subsection{Indirect search at LHC and future colliders}

With the global fit methods in Sect. 3, here we will utilize the SM-like Higgs precision measurement from LHC Run-II [31], HL-LHC [32] and CPEC [33]. In details, for LHC RunII we work with the ATLAS results ATLAS at $13 \mathrm{TeV}$ up to $80 \mathrm{fb}^{-1}$, and for HL-LHC, we work with combined results from future ATLAS and CMS, up to $6 a b^{-1}$. For CPEC, the latest designed luminosity is $5.6 a b^{-1}$ at $\sqrt{S}=240 \mathrm{GeV}$.

We give our global fit results in Fig. 1, the allowed region in the plane of $\tan \beta-\cos (\beta-\alpha)$ at $95 \%$ C.L. for the four types of 2HDM, given LHC Run-II (green), HL-LHC (blue) and CEPC (red) Higgs precision measurements. For the Type-I $2 \mathrm{HDM}$, all the SM-like Higgs fermion couplings are $\kappa_{U}$ type in Eq. (7) with $\cot \beta$-enhanced corrections when deviates from alignment limit $\cos (\beta-\alpha)=0$. Thus at large $\tan \beta$ region, the Yukawa couplings would not contribute much in constraining the parameter space, and the main restriction is from gauge couplings. The detailed values are displayed in Table 3.

For the other three types, they include both $\kappa_{U}$ and $\kappa_{D}$ type Yukawa couplings, as a result both large and small $\tan \beta$ are strongly constrained apart for the wrong-sign Yukawa regions. The relevant the maximally allowed $|\cos (\beta-\alpha)|$ ranges are also shown in Table 3. We also note the Type-LS is less restricted at small $\tan \beta$ compared to Type-II and Type$\mathrm{F}$, because only lepton couplings of Type-LS have $\kappa_{D}$ type and the precisions of $\delta \kappa_{b}$ is better than $\delta \kappa_{\tau}$, for example in CPEC, $\delta \kappa_{b}=1.3 \%, \delta \kappa_{\tau}=1.5 \%$.

The gray represent the wrong-sign Yukawa regions as Sect. 2.2, with $\kappa_{U} \kappa_{V}<0$ for Type-I, $\kappa_{b} \kappa_{V}<0$ for Type-II and Type-F, $\kappa_{\tau} \kappa_{V}<0$ for Type-II and Type-LS.

\subsection{Wrong-sign region and disappeared up-type}

From Eqs. (9) and (10), even $\cos (\beta-\alpha) \nRightarrow 0$ there are still allowed regions to get $\left|\kappa_{U, D}\right|=1$, which is the so called wrong-sign Yukawa region of $2 \mathrm{HDM}$ as defined (Eq. 12). As shown in Fig. 1, gray regions are of wrong-sign Yukawa couplings defined in Eq. (12). Since $\kappa_{V}>0$ keeps always, Eq. (12) means $\kappa_{U / D}<0$, with the lower left region for Type-I, and the upper right regions for Type-II/L/F. The later three types all have $\kappa_{U}$-type wrong-sign Yukawa region as Type-I, which are not shown out.

In details the $\kappa_{D}$-type wrong-sign Yukawa in Eq. (10) only occurs at $\tan \beta>1$. For the exact wrong-sign limit at tree level $\kappa_{U}=-\kappa_{D}=1, \sin (\beta-\alpha)=-\cos 2 \beta$, and at large $\tan \beta$, we have

$\cos (\beta-\alpha)=2 / \tan \beta$.

Thus even at CEPC, where we will have $\delta \kappa_{Z}=\mid 1-\sin (\beta-$ $\alpha) \mid \leq 0.25 \%$, the wrong-sign Yukawa is still allowed around $\cos (\beta-\alpha) \approx 2 / \tan \beta$ for $\cos (\beta-\alpha)<0.07$ at tree level.

$\kappa_{U}$-type wrong-sign Yukawa in Eq. (10) only occurs at $\tan \beta<1$. For the exact wrong-sign limit $\kappa_{D}=-\kappa_{U}=1$, $\sin (\beta-\alpha)=\cos 2 \beta$, and at small $\tan \beta$,

$\cos (\beta-\alpha)=-2 \tan \beta$.

Usually $\kappa_{U}$ and $\kappa_{D}$ are estimated in the form of $\kappa_{U, D}^{2}$, except for if there is any interference. The two sensitive parameters [32] are

$\kappa_{\gamma}=\left(1.59 \kappa_{W}^{2}-0.67 \kappa_{t} \kappa_{W}+0.071 \kappa_{t}^{2} \ldots\right)^{0.5}$,

$\kappa_{g}=\left(1.11 \kappa_{t}^{2}-0.12 \kappa_{t} \kappa_{b}+0.01 \kappa_{b}^{2} \ldots\right)^{0.5}$.

Here Eqs. (17) and (18) tell us the sign of $\kappa_{b}$ does not make an important enough difference to $\chi^{2}\left(\kappa_{b} \rightarrow 1\right)$ and $\chi^{2}\left(\kappa_{b} \rightarrow-1\right)$ through the global fit method Eq. (14) at tree level [35], while the sign of $\kappa_{t}$ makes an important difference to both $\kappa_{\gamma}, \kappa_{g}$. For $\kappa_{U}$-type wrong-sign region, corrected $\kappa_{\gamma \gamma}$ 

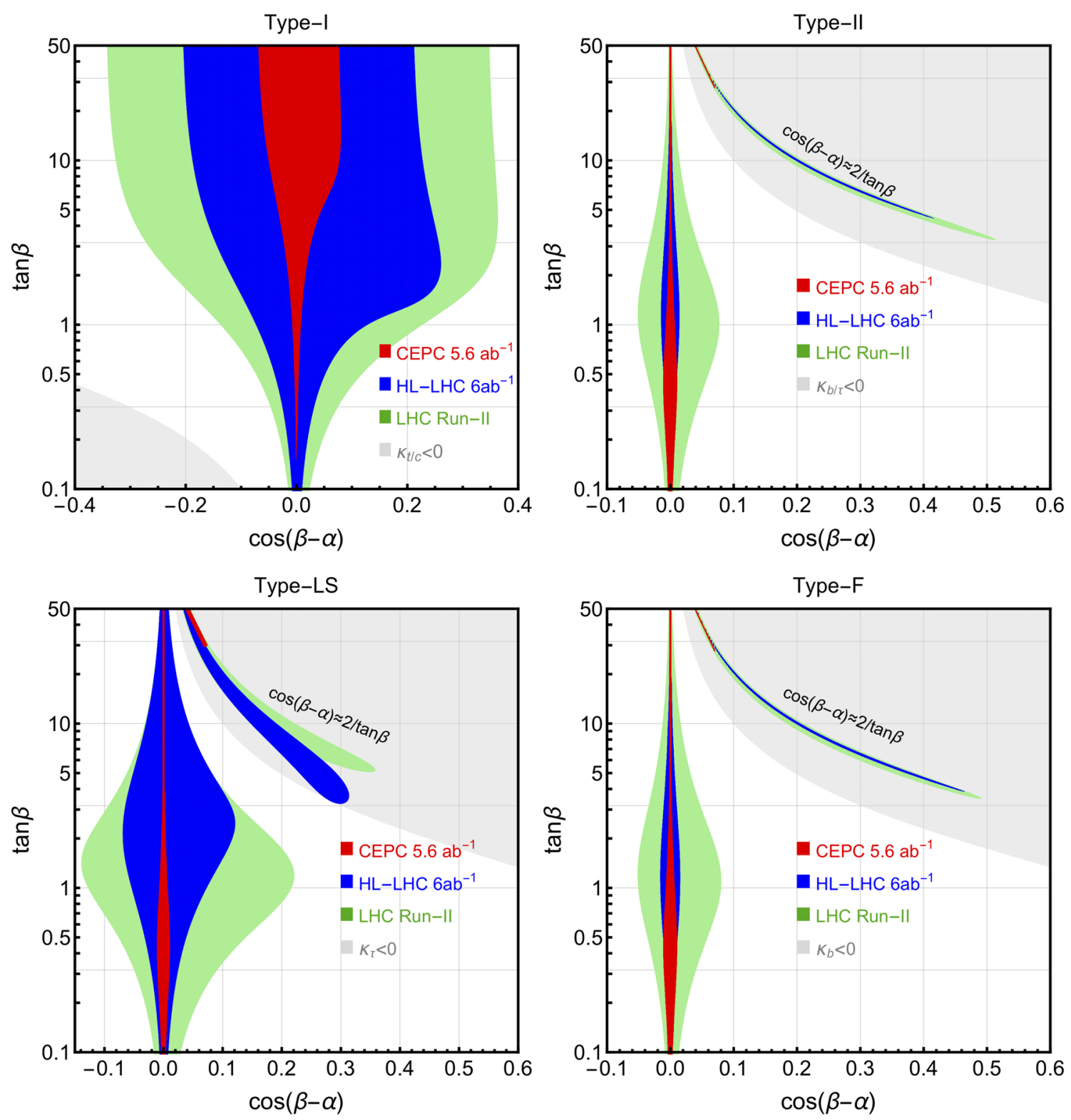

Fig. 1 The allowed region in the plane of $\tan \beta-\cos (\beta-\alpha)$ at $95 \%$ C.L. for the four types of 2HDM, given LHC Run-II (green), HL-LHC (blue) and CEPC (red) Higgs precision measurements. For future measurements, we assume that the measurements agree with SM predictions. The gray represent the wrong-sign Yukawa regions discussed at

Sect. 2.2, with $\kappa_{U} \kappa_{V}<0$ for Type-I, $\kappa_{b} \kappa_{V}<0$ for Type-II and Type$\mathrm{F}, \kappa_{\tau} \kappa_{V}<0$ for Type-II and Type-LS. . The colored "arm" regions for the Type-II, L and F are the allowed wrong-sign Yukawa regions correspondingly

deviated from SM values too large to be excluded. The colored "arm" regions for the Type-II, L and F are the allowed wrong-sign Yukawa regions correspondingly at 95\% C.L. under various Higgs precision measurements.

\subsection{Current LHC direct search}

Afte $r$ the indirect searches, here we will take the Type-

Table 3 Apart for the wrong-sign region, the maximally allowed $|\cos (\beta-\alpha)|$ range at 95\% C.L. given LHC Run-II, HL-LHC (including both ATLAS and CMS), and CEPC Higgs precision measurements

\begin{tabular}{llll}
\hline Type & LHC Run-II & HL-LHC & CEPC \\
\hline Type-I $\tan \beta \gtrsim 5$ & 0.38 & 0.2 & 0.08 \\
Type-II $\tan \beta \sim 1$ & 0.08 & 0.015 & 0.01 \\
Type-L $\tan \beta \sim 1$ & 0.22 & 0.12 & 0.011 \\
Type-F $\tan \beta \sim 1$ & 0.08 & 0.015 & 0.012 \\
\hline
\end{tabular}


searches, and to explore the combined constraint ability to the wrong-sign Yukawa region.

As shown in Fig. 2, the excluded region by current LHC direct search in the plane $m_{H / A}-\tan \beta$, including $A \rightarrow Z h(h \rightarrow b \bar{b})$ (red), $A / H \rightarrow b \bar{b}$ (purple), $H \rightarrow h h$ (cyan), $A / H \rightarrow \mu^{+} \mu^{-}$(yellow), $H \rightarrow V V$ (green), $A / H \rightarrow \tau^{+} \tau^{-}$(orange) respectively. Based one Fig. 1, to study the the wrong-sign Yukawa region, we take the benchmark parameter $\cos (\beta-\alpha)=0$ (left), 0.2 (middle), and 0.4 (right), with degenerate heavy Higgs mass $m_{A}=m_{H}$, $m_{H^{ \pm}}=\max \left\{600 \mathrm{GeV}, m_{H}\right\}, m_{H}^{2}=m_{12}^{2} / s_{\beta} c_{\beta}$. For the constraints from charged Higgs, on one hand, both the B-physics requiring $m_{H^{ \pm}}>580$ at $\tan \beta>0.7[44,45]$ and direct searches at LHC [46] do not have strong probe ability on wrong-sign Yukawa region as channels about heavy neutral ones. On the other hand, mass splittings between heavy Higgs are allowed [40]. Therefore charged Higgs constraints do not affect wrong-sign Yukawa regions from direct searches or affecting neutral heavy Higgs indirectly. After all in our studies, we take $m_{A}=m_{H}, m_{H^{ \pm}}=\max \left\{600 \mathrm{GeV}, m_{H}\right\}$.

In the left panel of Fig. 2, only $H / A \rightarrow f \bar{f}$ channels have constraint since $H h h, H V V, A h Z$ couplings at tree level are proportional to $\cos (\beta-\alpha)$. Generally the region $m_{A} \in(130,800), \tan \beta>10$ is excluded by $\tau \tau$ decay channel, and for larger heavy Higgs mass, the excluded $\tan \beta$ limit will be larger, to limitless around $1.5 \mathrm{TeV}$. Also a small region $m_{A} \in\left(130,2 m_{t}\right), \tan \beta \in(0.5,2)$ is excluded by $A / H \rightarrow \tau \tau$. For middle and right panels of Fig. 2, all channels here would make a difference with non-zero $\cos (\beta-\alpha)$. For $\cos (\beta-\alpha)=0.2$, at large $\tan \beta$ the regions of $m_{A}<700, \tan \beta>5, m_{A}<800, \tan \beta>10$ are excluded. Similarly the restriction ability goes down until $1.5 \mathrm{TeV}$. At small $\tan \beta$ region, $m_{A}<800, \tan \beta<0.3$ is strongly constrained. The excluded region can reach 1.2 $\mathrm{TeV}$ for $\tan \beta \in(0.9,2)$. For larger $\cos (\beta-\alpha)=0.4$, when $m_{A}<800, \tan \beta>3$ are strongly constrained since the more powerful $A \rightarrow Z h$ channel. This channel gets larger decay rates with larger $\cos (\beta-\alpha)$. But it can only reach $1.4 \mathrm{TeV}$ around $\tan \beta=30$. The excluded region of $\cos (\beta-\alpha)=0.4$ at small $\tan \beta$ region is similar as $\cos (\beta-\alpha)=0.2$. Another important feature is, the covered regions on $\tan \beta$ are nearly similar for $m_{A} \in\left(2 m_{t}, 800\right) \mathrm{GeV}$.

The strong constraints at large $\tan \beta$ and non-zero $\cos (\beta-$ $\alpha)$ can contribute to exclude the wrong-sign Yukawa region. To have a more straightforward idea, we will compare the direct and indirect searches in the plane $\cos (\beta-\alpha)-\tan \beta$.

As in Fig. 3, here we choose benchmark parameters $m_{A}=m_{H}=m_{H^{ \pm}}=800 \mathrm{GeV}$ (left and middle), 1500 $\mathrm{GeV}$ (right) and $\sqrt{\lambda v^{2}} \equiv \sqrt{\left(m_{H}^{2}-m_{12}^{2} / s_{\beta} c_{\beta}\right)}=100 \mathrm{GeV}$ (left and right), $600 \mathrm{GeV}$ (middle), to discuss the combine the constraint from indirect Higgs precision measurement and direct heavy Higgs searches at current LHC Run-II. The details about the experimental reports are same as Figs. 1 and 2. At left panel with $m_{A}=800 \mathrm{GeV}, \sqrt{\lambda v^{2}}=100 \mathrm{GeV}$ , the wrong-sign region at large $\tan \beta>20$ is totally covered by $A / H \rightarrow \tau \tau$ channel, and at small $\tan \beta$ region, it is strongly constrained by $A \rightarrow Z h$ channel. The small allowed region is around $8<\tan \beta<10,0.2<\cos (\beta-\alpha)<0.3$. At small $\tan \beta$ region, LHC direct searches give weak constraints resulting from too wide $\Gamma_{A / H}$ and current searches are not valid in this region. Compared the middle panel with $\sqrt{\lambda v^{2}}=600 \mathrm{GeV}$, the general results around wrong-sign Yukawa region are quite similar. This tells us the independence on $\sqrt{\lambda v^{2}}$ or $m_{12}$ in the considered regions. For the right panel with $m_{A}=1500 \mathrm{GeV}, \sqrt{\lambda v^{2}}=100 \mathrm{GeV}$, the LHC direct search can nearly give no constraints there, which is
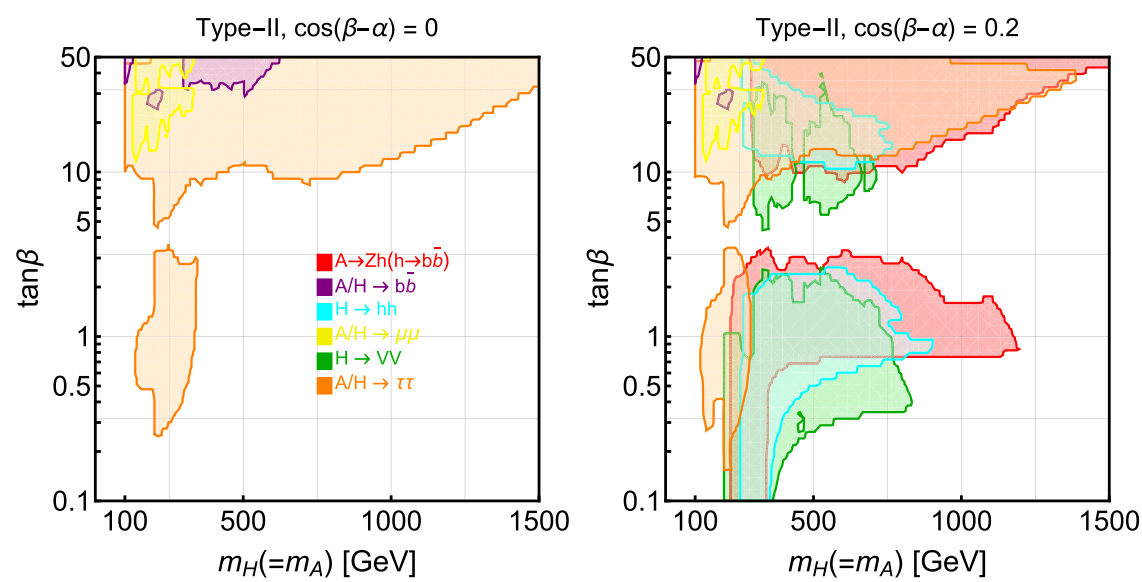

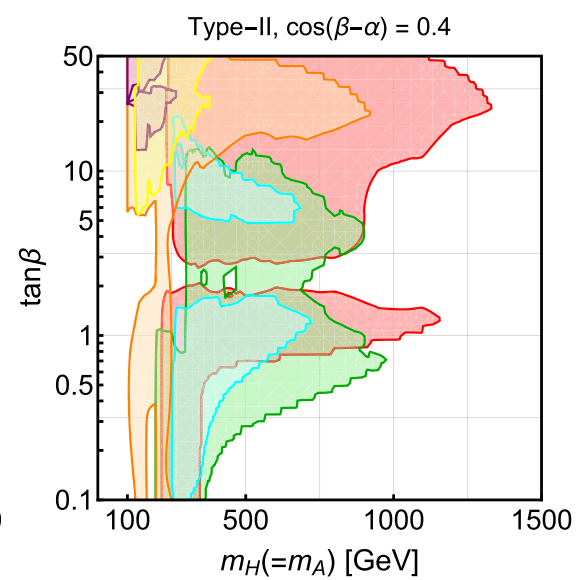

Fig. 2 Interpretation results of excluded region in the plane $m_{H / A}-$ $\tan \beta$ with latest LHC direct search limits, including $A \rightarrow$ $Z h(h \rightarrow b \bar{b})$ (red), $A / H \rightarrow b \bar{b}$ (purple), $H \rightarrow h h$ (cyan), $A / H \rightarrow \mu^{+} \mu^{-}$(yellow), $H \rightarrow V V$ (green), $A / H \rightarrow \tau^{+} \tau^{-}$ (orange). Here the benchmark parameter is degenerate heavy Higgs $\operatorname{mass} m_{A}=m_{H}, m_{H^{ \pm}}=\max \left\{600 \mathrm{GeV}, m_{H}\right\}, \quad \cos (\beta-$ $\alpha)=0$ (left), 0.2 (middle), 0.4 (right), and $m_{H}^{2}=m_{12}^{2} / s_{\beta} c_{\beta}$ 

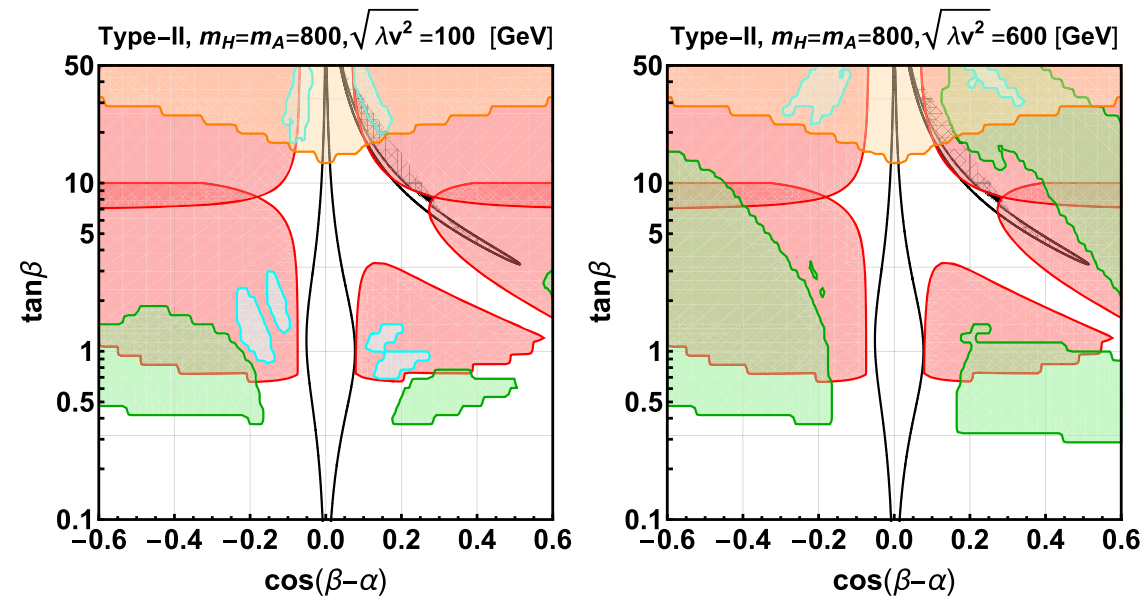

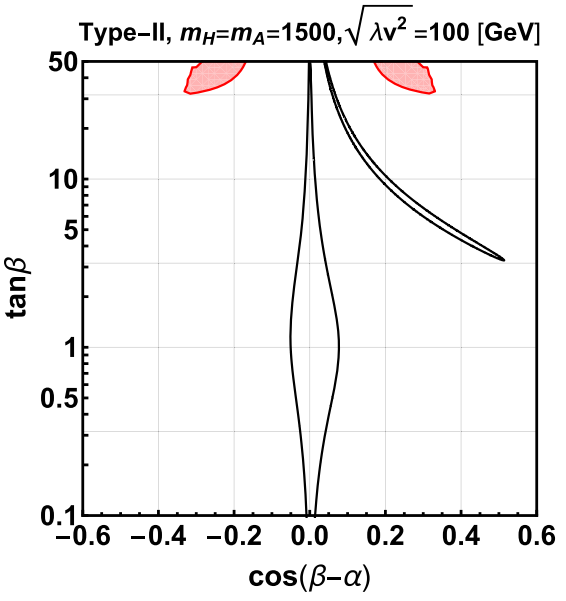

Fig. 3 Here we will show the excluded region from indirect Higgs precision measurements and direct search results in the plane $\tan \beta$ $\cos (\beta-\alpha)$ for Type II 2HDM. The indirect come from ATLAS at 13 $\mathrm{TeV}$ [31], and the direct seaches includs $A \rightarrow H Z$ (blue), $A \rightarrow Z h$ (red), $A / H \rightarrow \tau \tau$ (orange), $A / H \rightarrow b b$ (purple) and $A / H \rightarrow \mu \mu$ (yellow), to compare different channel constraining ability. Here we choose benchmark parameters $m_{A}=m_{H}=m_{H^{ \pm}}=800 \mathrm{GeV}$ (left and middle), $1500 \mathrm{GeV}$ (right) and $\sqrt{\lambda v^{2}} \equiv \sqrt{\left(m_{H}^{2}-m_{12}^{2} / s_{\beta} c_{\beta}\right)}=100$ $\mathrm{GeV}$ (left and right), $600 \mathrm{GeV}$ (middle). The current precision measurements results are shown by solid lines. Generally the central region between the two lines around $\cos (\beta-\alpha)=0$ are allowed except for the "arm" of Type-II, the wrong-sign Yukawa region as discussed detailed in Fig. 1 also shown in Fig. 2. Also from middle and right panels of Fig. 2, where the LHC direct search constraints are similar for $m_{A}<800 \mathrm{GeV}$ and large $\tan \beta$ region, we can say the wrong-sign region with $m_{A}<800 \mathrm{GeV}$ are strongly constrained by the combined indirect and direct searches at tree level.

For direct searches, channels like $H \rightarrow b b, \tau \tau$ can make differences if their couplings are $\tan \beta$-enhanced as from Table 2. For $H \tau \tau$ of Type-L, and $H b b$ of Type-F are also same as Type-II, and other channels are $\tan \beta$-reduced. Thus the constraints on wrong-sign region for Type-L/F would be same as Type-II, or weaker than Type-II.

\section{Results at one-loop level}

From last section, the combined indirect and direct searches at current LHC can give strong constraints on wrong-sign Yukawa region for $m_{A}<800 \mathrm{GeV}$ while for large heavy Higgs mass such $m_{A}=1500 \mathrm{GeV}$, direct searches nearly has no restrictions. The conclusion will be modified to a large extent when including the loop-level corrections to Higgs precision measurement study $[40,43]$.

\subsection{Loop effects in $\cos (\beta-\alpha)-\tan \beta$ plane}

To explore loop effects on the wrong-sign Yukawa region, here we first analyze the individual Higgs couplings cosntraints in details in Type-II 2HDM. In [40,43], we have detailed studies about the normal Yukawa regions around $\cos (\beta-\alpha)=0$, and the studies method here are similar, thus here we only display the wrong-sign regions.

As the Fig. 4, we show the allowed wrong-sign Yukawa region in the plane of $\tan \beta-\cos (\beta-\alpha)$ at $95 \%$ C.L. for TypeII 2HDM, given LHC Run-II Higgs precision measurements at one-loop level. The benchmark parameters in the left panel is $m_{A}=m_{H}=m_{H^{ \pm}}=800 \mathrm{GeV}, \lambda v^{2}=-100^{2}$. The gray regions are of $\kappa_{b}<0$ as in Fig. 1. The blue region is allowed at one-loop level, and the red and green lines are for $\delta \kappa_{b}= \pm 0.19$ and $\delta \kappa_{Z}= \pm 0.08$ taken from current LHC reports [32].

The allowed region by $h Z Z$ coupling at one-loop level are always around $\cos (\beta-\alpha)=0$ displayed by green line, similar to tree level. For $h b b$ the case becomes parameter dependent, with $\kappa_{b}=-1 \pm 0.19$ represented by red lines. In the left panel with $\lambda v^{2}=-100^{2} \mathrm{GeV}^{2}$, region with $\kappa_{b}<0$ gets reduced compared to it at tree level, as well as the allowed wrong-sign Yukawa "arm". In the middle panel with $\lambda v^{2}=0 \mathrm{GeV}^{2}$, the upper right regions has $\kappa_{b}>0$, resulting to two regions of $\kappa_{b}=-1 \pm 0.19$. For right panel with $\lambda v^{2}=600^{2} \mathrm{GeV}^{2}, \kappa_{b}<0$ region gets larger, and the allowed wrong-sign Yukawa "arm" shifts a lot compared to themselves at tree level. Generally we can conclude, the blue allowed wrong-sign Yukawa regions are mainly dependent on $h b b, h Z Z$ channels at one-loop level at Type-II.

In Fig. 5, based on the analysis in Fig. 4, we show the allowed wrogn-sign Yukawa regions of various $\lambda v^{2}$ values at one-loop level in the plane of $\tan \beta-\cos (\beta-\alpha)$ at 95\% C.L. for Type-II 2HDM, given LHC Run-II Higgs precision measurements at one-loop level. Here we work with the bench- 

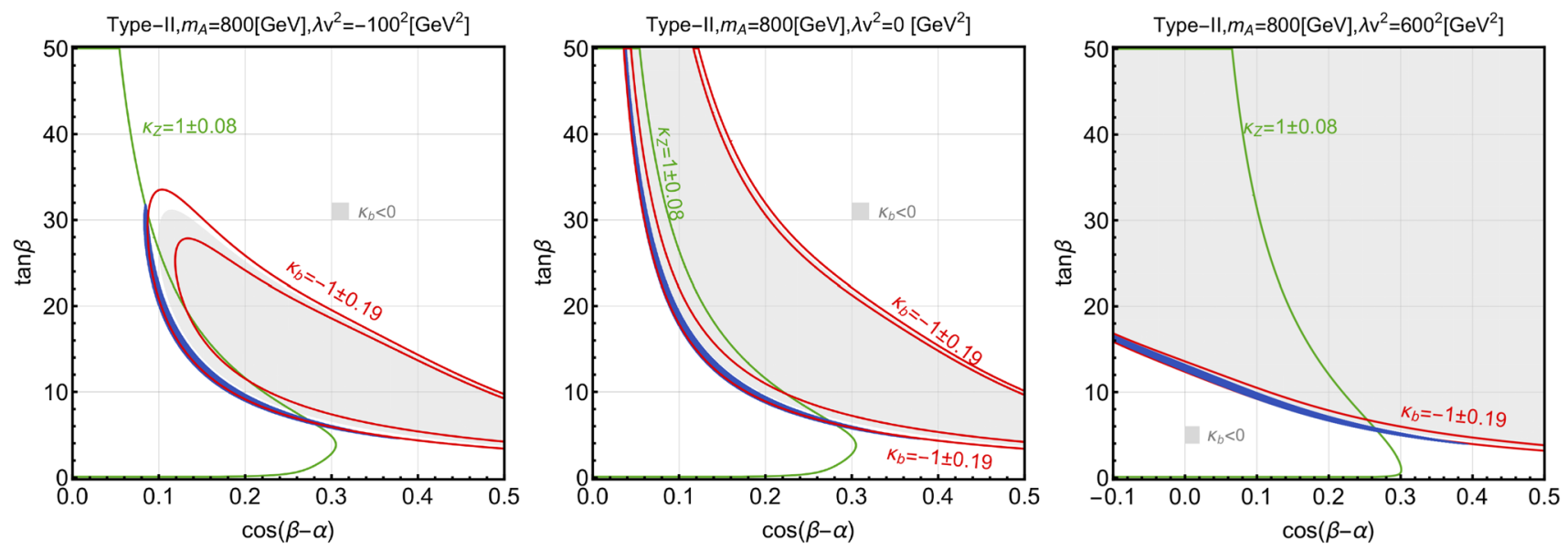

Fig. 4 The blue allowed region in the plane of $\tan \beta-\cos (\beta-\alpha)$ at 95\% C.L. for Type-II 2HDM, given LHC Run-II Higgs precision measurements at one-loop level. Here we take the benchmark parameters $m_{A}=m_{H}=m_{H^{ \pm}}=800 \mathrm{GeV}, \lambda v^{2}=-100^{2}$ (left), 0 (middle), abd

$600^{2}$ (right) $\mathrm{GeV}^{2}$. The gray regions are of $\kappa_{b}<0$ as in Fig. 1. We also show the current precision $\delta \kappa_{b}= \pm 0.19$ and $\delta \kappa_{Z}= \pm 0.08$ with red and green lines respectively, whose overlap parts are blue allowed regions

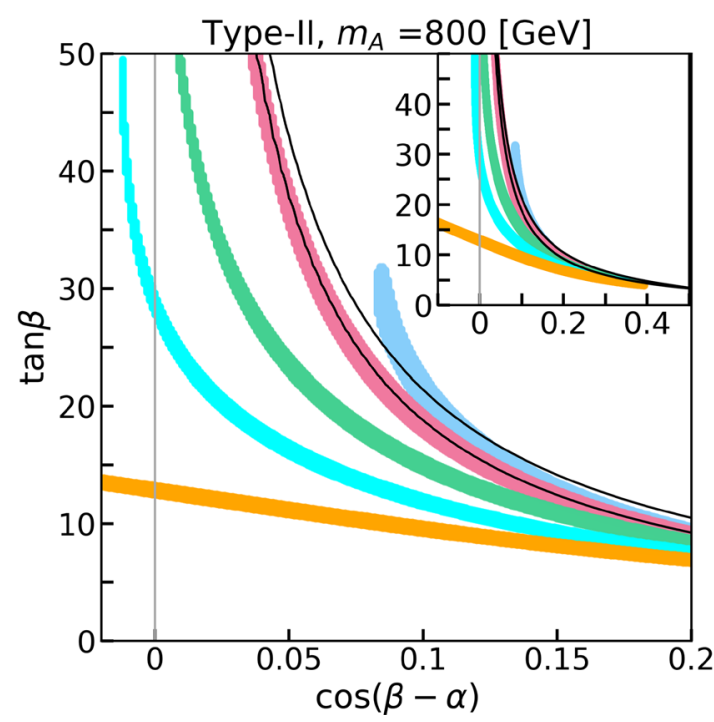

Fig. 5 The summarized allowed wrogn-sign Yukawa region in the plane of $\tan \beta-\cos (\beta-\alpha)$ at 95\% C.L. for Type-II 2HDM, given LHC Run-II Higgs precision measurements at one-loop level. Here we take the benchmark parameters $m_{A}=m_{H}=m_{H^{ \pm}}=800 \mathrm{GeV}$ (left) and $1500 \mathrm{GeV}$ (right). The diffenet colorful regions are for $\lambda v^{2}=-100^{2}$

mark parameters $m_{A}=m_{H}=m_{H^{ \pm}}=800 \mathrm{GeV}$ (left), 1500 $\mathrm{GeV}$ (right), $\lambda v^{2}=-100^{2}, 0,50^{2}, 200^{2}, 400^{2}, 600^{2} \mathrm{GeV}^{2}$ displayed by blue, light red, magenta, green and cyan regions respectively. At the left panel, the main plot shows $\cos (\beta-$ $\alpha) \in(-0.02,0.2)$, and the larger region is in the subplot for $\cos (\beta-\alpha) \in(-0.02,0.4)$. The allowed wrong-sign Yukawa region at tree level is displayed by black solid lines. Here the light red region for $\lambda v^{2}=0$ is the most similar one to the tree level region, and regions of smaller $\lambda v^{2}$ would locate at right while regions of larger $\lambda v^{2}$ would shift to

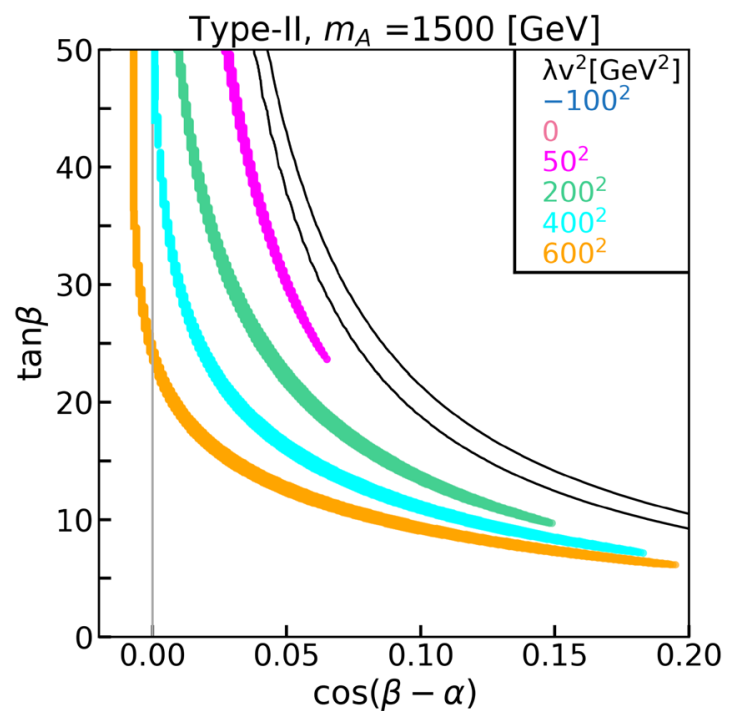

(blue), 0 (light red), $50^{2}$ (magenta), $200^{2}$ (green), $400^{2}$ (cyan) and $600^{2}$ (orange) $\mathrm{GeV}^{2}$. We also show the allowed wrong-sign Yukawa region at tree level with black solid lines. For $m_{A}=800 \mathrm{GeV}$, we show the larger allowed region in the subplot, upper right corner of the left panel

the left of black lines. Thus this range would be less constrained by current LHC direct searches as shown in Fig. 3 . For $m_{A}=1500 \mathrm{GeV}$, region of $\lambda v^{2} \leq 0$ is totally excluded, and for large $\lambda v^{2}$ the allowed region is shifted to the left of black lines as $m_{A}=800 \mathrm{GeV}$. Here we also see, the allowed $\cos (\beta-\alpha)$ range at loop level is larger than it at tree level, for both cases. Usually theoretical constraints can restrict large $\left|\lambda v^{2}\right|$ strongly, especially for $\sqrt{\left|\lambda v^{2}\right|}>100$ $\mathrm{GeV}$, while Higgs precision measurements is complementary on constraining small $\lambda v^{2}$ for large mass. 


\subsection{Loop effects in $m_{\Phi}-m_{12}$ plane}

Since there are weak theoretical constraints around $\lambda v^{2}=$ $m_{H / A}^{2}-m_{12}^{2} /(\sin \beta \cos \beta)=0$, here we explore this special region carefully, in the plane of $m_{A}-m_{12}$.

In Fig. 6, performing the global fit at 95\% C.L. for TypeII 2HDM, we show the allowed region in the plane of $m_{A}$ - $m_{12}$ after including the loop corrections to SM-like Higgs couplings. For the benchmark parameters, we still take heavy Higgs mass $m_{A}=m_{H}, m_{H^{ \pm}}=600 \mathrm{GeV}$, with $\cos (\beta-$ $\alpha)=0.05$ (left), 0.07 (right), $\tan \beta=30$ (blue), 35 (green), 45 (red). The global fit results with current LHC and future HLLHC Higgs precision measurements are displayed with light and dark colors respectively. With the future CEPC reports, the allowed region is strongly constrained, and since the $\chi^{2}$ of best point is larger than 100 for these cases, we would not show them here.

Generally for a pair of fixed $\cos (\beta-\alpha)$ and $\tan \beta$, the allowed wrong-sign Yukawa regions at one-loop level are divided into two parts based on $\tan \beta=20$. For $m_{12}>20 \mathrm{GeV}$, the allowed region tends to have $\sqrt{\lambda v^{2}}=$ $\sqrt{m_{A}^{2}-m_{12}^{2}\left(1+\tan ^{2} \beta\right) / \tan \beta} \approx 0 \mathrm{GeV}$, where there is weak constraints from theory, and $m_{A}<(1.5-2) \mathrm{TeV}$. Larger $m_{A}$ range is excluded because of too large loop corrections. In the plots, we also show the dashed line indicating the tiny regions allowed by theoretical constraints for corresponding parameters. The allowed region partially are same as the colored region allowed by Higgs precision measurements. For $m_{12}<20 \mathrm{GeV}$, the allowed region has large

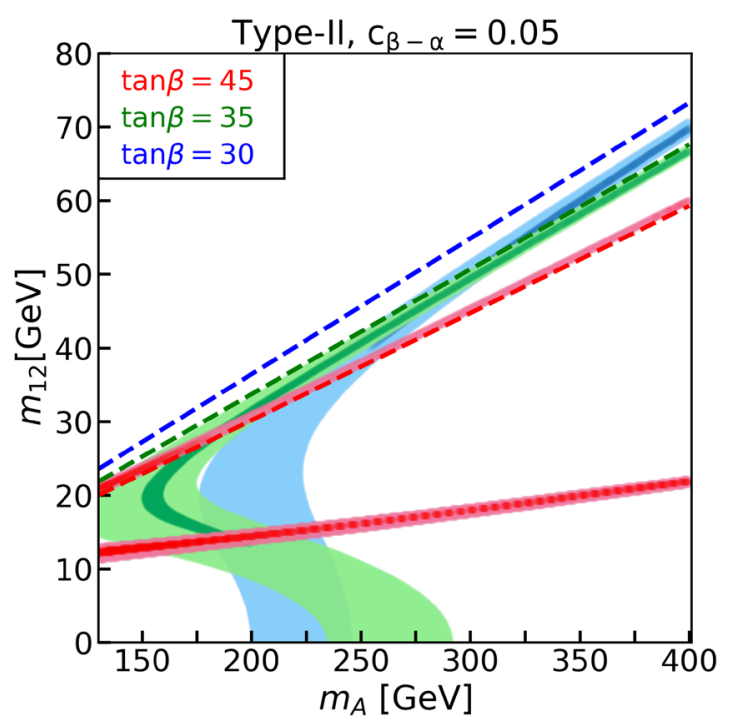

Fig. 6 The allowed region in the plane of $m_{A}-m_{12}$ at 95\% C.L. for Type-II 2HDM, given LHC Run-II (light color) and HL-LHC (dark color) Higgs precision measurements at one-loop level. Here we take the benchmark parameters $m_{A}=m_{H}, m_{H^{ \pm}}=600 \mathrm{GeV}, \cos (\beta-\alpha)=$ 0.05 (left), 0.07 (right), $\tan \beta=30$ (blue), 35 (green), 45 (red). Gener- $\left|\sqrt{\lambda v^{2}}\right|>100 \mathrm{GeV}$, to excluded by theoretical constraints [40]. Therefore we can conclude, constraints from Higgs precision measurements works better than theoretical constraints at small $\lambda v^{2}$, and the two together could constrain the whole $\lambda v^{2}$ stronger.

Based on Eq. (15), the wrong-sign Yukawa region at tree level has a simple relationship $\cos (\beta-\alpha) \approx 2 / \tan \beta$ when $\tan \beta \gg 1$. This relationship at one-loop level would not keep anymore, since for a specific $\cos (\beta-\alpha)$, different $\tan \beta$ s are allowed.

\section{Conclusions}

Since the discovery of SM-like Higgs boson at LHC Run-I, exploring its properties especially Higgs couplings become a promising method to study new physics. In the framework of 2HDM, this work focuses on testing the so-called wrongsign Yukawa region up to one-loop level. It is known that wrong-sign limit of Type-II is $\kappa_{D}=-1$, and $\kappa_{U}=1$. $\sin (\beta+\alpha)=1$ can reach it at tree level. We pointed out that, the limit at one-loop level requires heavy Higgs mass decoupling as well.

Our study worked with both indirect and direct searches at current LHC, to search the region before decoupling scale. For the direct searches, we constrained the parameter space with various heavy Higgs decays, $A / H \rightarrow f \bar{f}, V V, V h, h h$ at tree level. For the indirect searches, we perform the global

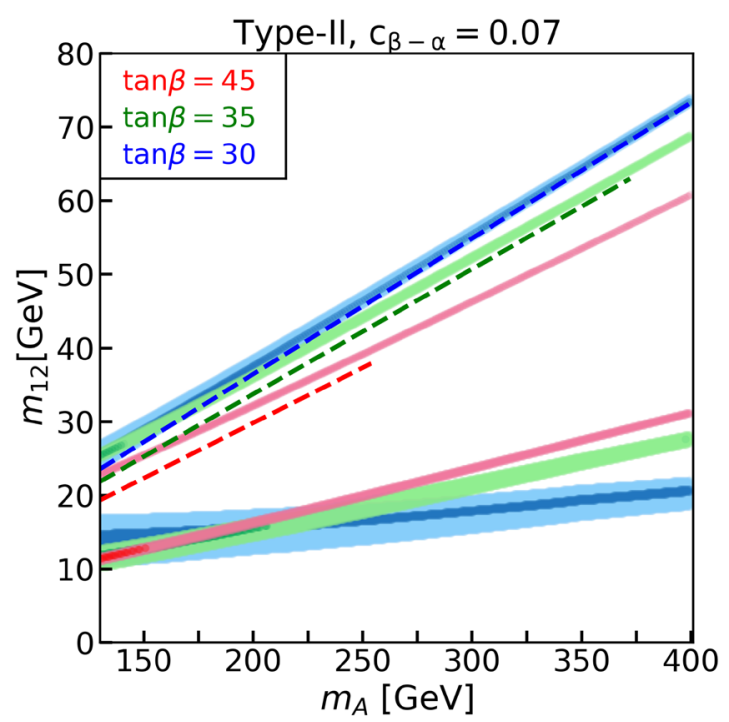

ally for a pair of fixed $\cos (\beta-\alpha)$ and $\tan \beta$, the allowed wrong-sign Yukawa region tends to get a linear relationship between $m_{A}-m_{12}$, until $m_{A}$ approaches (1.5-2) TeV to be excluded by current LHC Run-II data. The dashed line are allowed line (tiny region) by theoretical constraints 
fit with current LHC, future HL-LHC and CEPC Higgs precision measurements up to one-loop level.

Generally as shown in Figs. 1, 2, 3, for heavy Higgs mass $m_{A}=m_{H}<800 \mathrm{GeV}, m_{H^{ \pm}}=\max \left\{m_{H}, 600 \mathrm{GeV}\right\}$, the wrong-sign Yukawa regions at tree level are excluded largely for Type-II 2HDM, except for the tiny allowed region around $\tan \beta \in(8,10)$ under the combined direct and indirect searches of current LHC data at tree level. The excluded region is also nearly independent of parameter $m_{12}$ or $\lambda v^{2}=$ $m_{A}^{2}-m_{12}^{2} /(\sin \beta \cos \beta)$. For larger $m_{A}$, the constraints get weaker, and direct searches can not put any more constraints on the wrong-sign region for $m_{A}=1500 \mathrm{GeV}$.

The excluded region would change much after including loop corrections to the indirect Higgs precison measuremetns studies. Comparing Fig. 1 and Fig. 4, the $\operatorname{sign}\left(\kappa_{b}\right)=-1$ region and the allowed wrong-sign Yukawa region could be corrected magnificently in some parameter space, which is mainly depedent on $h b b, h Z Z$ channels for Type-II. Unlike the results at the tree level, $m_{12}$ or $\lambda v^{2}$ could also make a difference. From Fig. 5, we can conclude that the wrongsign region with $\lambda v^{2}>0$ will be less constrained by heavy Higgs direct searches at Fig. 2 for small mass such as $m_{A}=$ $800 \mathrm{GeV}$. For large mass, such as our case study with $m_{A}=$ $1500 \mathrm{GeV}$ where is no constraints from direct searches at tree level, region of $\lambda v^{2} \leq 0$ is totally excluded, and for large $\lambda v^{2}>50 \mathrm{GeV}^{2}$ the allowed region is shifted to the left of the tree-level region. In general we can conclude that with loop corrections, wrong-sign Yukawa regions of small $\lambda v^{2}$ will be more constrained, while the range of large $\lambda v^{2}$ is less constrained under current LHC direct and indirect limits. These features are quite different to the results at tree level. Since theoretical constraints put weak restriction on small $\left|\lambda v^{2}\right|$, at Fig. 6 we explored the $\lambda v^{2}=m_{A}^{2}-m_{12}^{2} /(\sin \beta \cos \beta) \approx 0$ $\mathrm{GeV}$. We found Higgs measurements works better here than theoretical constraints. There are still allowed regions under current LHC form $m_{A}<1500 \mathrm{GeV}$, but when considering the future CEPC, it is difficult to find out the survived points.

Acknowledgements We thank Huayang Song for useful discussions. This work is supported by the Australian Research Council Discovery Project DP180102209.

Data Availability Statement This manuscript has no associated data or the data will not be deposited. [Authors' comment: The work has no data to be deposited. All the important results and formulae are included in the main draft.]

Open Access This article is licensed under a Creative Commons Attribution 4.0 International License, which permits use, sharing, adaptation, distribution and reproduction in any medium or format, as long as you give appropriate credit to the original author(s) and the source, provide a link to the Creative Commons licence, and indicate if changes were made. The images or other third party material in this article are included in the article's Creative Commons licence, unless indicated otherwise in a credit line to the material. If material is not included in the article's Creative Commons licence and your intended use is not permitted by statutory regulation or exceeds the permitted use, you will need to obtain permission directly from the copyright holder. To view a copy of this licence, visit http://creativecomm ons.org/licenses/by/4.0/.

Funded by SCOAP ${ }^{3}$.

\section{References}

1. ATLAS Collaboration, G. Aad et al. Observation of a new particle in the search for the Standard Model Higgs boson with the ATLAS detector at the LHC. Phys. Lett. B 716, 1-29 (2012). arXiv: 1207.7214 [hep-ex]

2. C.M.S. Collaboration, S. Chatrchyan et al., Observation of a new boson at a mass of $125 \mathrm{GeV}$ with the CMS experiment at the LHC. Phys. Lett. B 716, 30-61 (2012). arXiv:1207.7235 [hep-ex]

3. J. Bernon, J.F. Gunion, H.E. Haber, Y. Jiang, S. Kraml, Scrutinizing the alignment limit in two-Higgs-doublet models: $\mathrm{m}_{h}=125 \mathrm{GeV}$. Phys. Rev. D 92(7), 075004 (2015). arXiv:1507.00933 [hep-ph]

4. J. Bernon, J.F. Gunion, H.E. Haber, Y. Jiang, S. Kraml, Scrutinizing the alignment limit in two-Higgs-doublet models. II. $\mathrm{m}_{H}=125 \mathrm{GeV}$. Phys. Rev. D 93(3), 035027 (2016). arXiv:1511.03682 [hep-ph]

5. E.J. Chun, The muon g-2 in two Higgs doublet models. EPJ Web Conf. 118, 01006 (2016). arXiv:1511.05225 [hep-ph]

6. L. Wang, X.-F. Han, A light pseudoscalar of 2HDM confronted with muon g-2 and experimental constraints. JHEP 05, 039 (2015). arXiv:1412.4874 [hep-ph]

7. P.M. Ferreira, R. Guedes, J.F. Gunion, H.E. Haber, M.O.P. Sampaio, R. Santos, The Wrong Sign limit in the 2HDM. in Proceedings, 2nd Conference on Large Hadron Collider Physics Conference (LHCP 2014): New York, USA, June 2-7, 2014 (2014). arXiv:1410.1926 [hep-ph]. http://www.slac.stanford.edu/ econf/C140602.2/papers/1410.1926v1.pdf

8. D. Fontes, J.C. Romão, J.P. Silva, A reappraisal of the wrong-sign $h b \bar{b}$ coupling and the study of $h \rightarrow Z \gamma$. Phys. Rev. D 90(1), 015021 (2014). arXiv: 1406.6080 [hep-ph]

9. P.M. Ferreira, R. Guedes, M.O.P. Sampaio, R. Santos, Wrong sign and symmetric limits and non-decoupling in 2HDMs. JHEP 12, 067 (2014). arXiv:1409.6723 [hep-ph]

10. A. Biswas, A. Lahiri, Alignment, reverse alignment, and wrong sign Yukawa couplings in two Higgs doublet models. Phys. Rev. D 93(11), 115017 (2016). arXiv:1511.07159 [hep-ph]

11. N. Greiner, S. Liebler, G. Weiglein, Interference contributions to gluon initiated heavy Higgs production in the Two-Higgs-Doublet Model. Eur. Phys. J. C 76(3), 118 (2016). arXiv:1512.07232 [hep$\mathrm{ph}]$

12. T. Modak, J.C. Romão, S. Sadhukhan, J.P. Silva, R. Srivastava, Constraining wrong-sign $h b b$ couplings with $h \rightarrow \Upsilon \gamma$. Phys. Rev. D 94(7), 075017 (2016). arXiv:1607.07876 [hep-ph]

13. P.M. Ferreira, S. Liebler, J. Wittbrodt, $p p \rightarrow A \rightarrow Z h$ and the wrong-sign limit of the two-Higgs-doublet model. Phys. Rev. D 97(5), 055008 (2018). arXiv:1711.00024 [hep-ph]

14. L. Wang, R. Shi, X.-F. Han, Wrong sign Yukawa coupling of the 2HDM with a singlet scalar as dark matter confronted with dark matter and Higgs data. Phys. Rev. D 96(11), 115025 (2017). arXiv:1708.06882 [hep-ph]

15. N.M. Coyle, B. Li, C.E.M. Wagner, Wrong sign bottom Yukawa coupling in low energy supersymmetry. Phys. Rev. D 97(11), 115028 (2018). arXiv:1802.09122 [hep-ph]

16. C.-W. Chiang, K. Yagyu, Implications of Higgs boson search data on the two-Higgs doublet models with a softly broken $Z_{2}$ symmetry. JHEP 07, 160 (2013). arXiv:1303.0168 [hep-ph]

17. CMS Collaboration, C. Collaboration, A Search for Beyond Standard Model Light Bosons Decaying into Muon Pairs 
18. CMS Collaboration, A.M. Sirunyan et al., Search for MSSM Higgs bosons decaying to $\mu^{+} \mu^{-}$in proton-proton collisions at $\sqrt{s}=13$ TeV. arXiv:1907.03152 [hep-ex]

19. ATLAS Collaboration, M. Aaboud et al., Search for scalar resonances decaying into $\mu^{+} \mu^{-}$in events with and without $b$-tagged jets produced in proton-proton collisions at $\sqrt{s}=13 \mathrm{TeV}$ with the ATLAS detector. JHEP (2019). arXiv:1901.08144 [hep-ex]

20. C.M.S. Collaboration, A.M. Sirunyan et al., Search for beyond the standard model Higgs bosons decaying into a $b \bar{b}$ pair in $p p$ collisions at $\sqrt{s}=13$ TeV. JHEP 08, 113 (2018). arXiv:1805.12191 [hep-ex]

21. ATLAS Collaboration, G. Aad et al., Search for heavy neutral Higgs bosons produced in association with $b$-quarks and decaying to $b$-quarks at $\sqrt{s}=13 \mathrm{TeV}$ with the ATLAS detector. arXiv:1907.02749 [hep-ex]

22. CMS Collaboration, C. Collaboration, Search for additional neutral MSSM Higgs bosons in the di-tau final state in $p p$ collisions at $\sqrt{s}=13 \mathrm{TeV}$

23. C.M.S. Collaboration, A.M. Sirunyan et al., Search for additional neutral MSSM Higgs bosons in the $\tau \tau$ final state in proton-proton collisions at $\sqrt{s}=13$ TeV. JHEP 09, 007 (2018). arXiv: 1803.06553 [hep-ex]

24. ATLAS Collaboration, M. Aaboud et al., Search for additional heavy neutral Higgs and gauge bosons in the ditau final state produced in $36 \mathrm{fb}^{-1}$ of pp collisions at $\sqrt{s}=13 \mathrm{TeV}$ with the ATLAS detector. JHEP 01, 055 (2018). arXiv:1709.07242 [hep-ex]

25. CMS Collaboration, A. M. Sirunyan et al., Search for heavy Higgs bosons decaying to a top quark pair in proton-proton collisions at $\sqrt{s}=13$ TeV. arXiv:1908.01115 [hep-ex]

26. ATLAS Collaboration, M. Aaboud et al., Search for heavy particles decaying into a top-quark pair in the fully hadronic final state in $p p$ collisions at $\sqrt{s}=13 \mathrm{TeV}$ with the ATLAS detector. Phys. Rev. D 99(9), 092004 (2019). arXiv:1902.10077 [hep-ex]

27. C.M.S. Collaboration, A.M. Sirunyan et al., Search for a new scalar resonance decaying to a pair of $Z$ bosons in proton-proton collisions at $\sqrt{s}=13$ TeV. JHEP 06, 127 (2018). arXiv: 1804.01939 [hep-ex]

28. ATLAS Collaboration, M. Aaboud et al., Search for heavy ZZ resonances in the $\ell^{+} \ell^{-} \ell^{+} \ell^{-}$and $\ell^{+} \ell^{-} v \bar{v}$ final states using protonproton collisions at $\sqrt{s}=13 \mathrm{TeV}$ with the ATLAS detector. Eur. Phys. J. C 78(4), 293 (2018). arXiv:1712.06386 [hep-ex]

29. CMS Collaboration, C. Collaboration, Search for high mass Higgs to WW with fully leptonic decays using 2015 data,

30. ATLAS Collaboration, M. Aaboud et al., Search for heavy resonances decaying into $W W$ in the $e \nu \mu \nu$ final state in $p p$ collisions at $\sqrt{s}=13 \mathrm{TeV}$ with the ATLAS detector. Eur. Phys. J. C 78(1), 24 (2018). arXiv:1710.01123 [hep-ex]

31. ATLAS Collaboration, T. A. collaboration, Combined measurements of Higgs boson production and decay using up to $80 \mathrm{fb}^{-1}$ of proton-proton collision data at $\sqrt{s}=13 \mathrm{TeV}$ collected with the ATLAS experiment
32. HL/HE WG2 group Collaboration, M. Cepeda et al., Higgs Physics at the HL-LHC and HE-LHC. arXiv:1902.00134 [hep-ph]

33. CEPC Study Group Collaboration, M. Dong et al., CEPC Conceptual Design Report: Volume 2 - Physics \& Detector. arXiv:1811.10545 [hep-ex]

34. G.C. Branco, P.M. Ferreira, L. Lavoura, M.N. Rebelo, M. Sher, J.P. Silva, Theory and phenomenology of two-Higgs-doublet models. Phys. Rept. 516, 1-102 (2012). arXiv:1106.0034 [hep-ph]

35. P.M. Ferreira, J.F. Gunion, H.E. Haber, R. Santos, Probing wrongsign Yukawa couplings at the LHC and a future linear collider. Phys. Rev. D 89(11), 115003 (2014). arXiv:1403.4736 [hep-ph]

36. S. Liebler, S. Patel, G. Weiglein, Phenomenology of on-shell Higgs production in the MSSM with complex parameters. Eur. Phys. J. C 77(5), 305 (2017). arXiv:1611.09308 [hep-ph]

37. D. Eriksson, J. Rathsman, O. Stal, 2HDMC: Two-Higgs-Doublet model calculator physics and manual. Comput. Phys. Commun. 181, 189-205 (2010). arXiv:0902.0851 [hep-ph]

38. A. Denner, Techniques for calculation of electroweak radiative corrections at the one loop level and results for W physics at LEP-200. Fortsch. Phys. 41, 307-420 (1993). arXiv:0709.1075 [hep-ph]

39. S. Kanemura, Y. Okada, E. Senaha, C.-P. Yuan, Higgs coupling constants as a probe of new physics. Phys. Rev. D 70, 115002 (2004). arXiv:hep-ph/0408364

40. N. Chen, T. Han, S. Su, W. Su, Y. Wu, Type-II 2HDM under the precision measurements at the $Z$-pole and a Higgs Factory. JHEP 03, 023 (2019). arXiv:1808.02037 [hep-ph]

41. S. Kanemura, M. Kikuchi, K. Sakurai, K. Yagyu, H-COUP: a program for one-loop corrected Higgs boson couplings in nonminimal Higgs sectors. Comput. Phys. Commun. 233, 134-144 (2018). arXiv:1710.04603 [hep-ph]

42. F.C.C. Collaboration, A. Abada et al., FCC-ee: The Lepton Collider. Eur. Phys. J. ST 228(2), 261-623 (2019)

43. J. Gu, H. Li, Z. Liu, S. Su, W. Su, Learning from Higgs physics at future higgs factories. JHEP 12, 153 (2017). arXiv:1709.06103 [hep-ph]

44. HFLAV Collaboration, Y. Amhis et al., Averages of $b$-hadron, $c$ hadron, and $\tau$-lepton properties as of summer 2016. Eur. Phys. J. C 77(12), 895 (2017). arXiv:1612.07233 [hep-ex]

45. F. Arco, S. Heinemeyer, M. Herrero, Exploring sizable triple Higgs couplings in the 2HDM. arXiv:2005.10576 [hep-ph]

46. S. Li, H. Song, S. Su, Probing exotic charged higgs decays in the type-II 2HDM through Top Rich Signal at a Future $100 \mathrm{TeV}$ pp Collider. arXiv:2005.00576 [hep-ph] 